\title{
Homogenization of Lamination Stacks in Linear Magnetodynamics
}

\author{
Laurent Krähenbühl, Patrick Dular, Tarek Zeidan, and François Buret
}

\begin{abstract}
An effective numerical modeling of devices with lamination stacks cannot be done without replacing the laminations by an equivalent solid region. So far, no convincing model has been proposed, even in the "simple" case of linear magnetodynamics-particularly if the resultant current in each sheet does not cancel. In this paper, we define the homogenized fields, and the related material properties. The noteworthy precision of the theoretic homogenized solution is shown and discussed by comparing it with the exact solution of the problem with laminations in the case of a simple "1.5-D" analytical test problem. The three-dimensional finite element solutions-real description of the sheets, refined mesh/homogenized region, coarser mesh-for the same test problem show also a perfect agreement.
\end{abstract}

Index Terms-Eddy currents, finite element methods, homogenization, lamination stack, magnetodynamics.

\section{INTRODUCTION}

$\mathbf{T}$ WO papers published over the last three years propose new ideas concerning the homogenization of lamination stacks, to lead to simplified but accurate models for finite elements. In [1] a static anisotropic problem is first solved, then the eddy currents are processed as a second-order effect. The analytical space and time changes of fields and current are correctly taken into account; however, this method cannot be applied if the eddy currents have a perceptible effect on the static solution. Very recently in [2], the same analytical one-dimensional (1-D) solutions have been a priori integrated into the test functions of the finite element (FE) formulation. The results obtained in this way are valid for a very large frequency range; on the other hand, only situations with a symmetrical magnetic flux density distribution in the normal direction of the iron sheets (i.e., with an odd distribution of the current density) have been taken into account. In this paper, the validity of these formulations is extended.

\section{HOMOGENIZATION}

\section{A. Analytical Form Functions and Characteristic Function $\underline{\mathrm{G}}$}

The well-known analytical solutions in a linear and conducting iron sheet concern the values of the tangential components of the magnetic flux density and magnetic field (in a direction $\alpha$ ) on one hand, of the current density and electric field (in the direction $\beta$ perpendicular to $\alpha$ ) on the other hand. All these quantities vary in the direction $\gamma$ normal to the sheets,

Manuscript received July 1, 2003.

L. Krähenbühl, T. Zeidan, and F. Buret are with CEGELY, UMR CNRS 5005, École Centrale de Lyon, F-69134 Écully Cedex, France.

P. Dular is with the Department of Electrical Engineering, University of Liège, B-4000 Liège, Belgium, and with the Belgian National Fund for Scientific Research (e-mail: Patrick.Dular@ulg.ac.be).

Digital Object Identifier 10.1109/TMAG.2004.825435

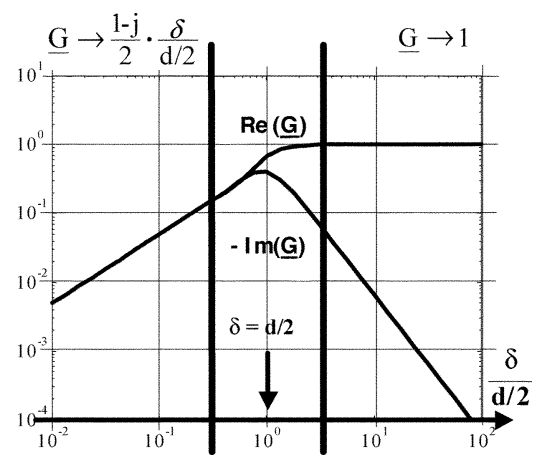

Fig. 1. Characteristic function $\mathrm{G}$ as a function of the relative skin depth, and its asymptotic values.

following the same shape functions $\underline{f}(\gamma)$ et $g(\gamma)$, which are, respectively, odd and even functions:

$$
\begin{aligned}
& \underline{\mathrm{f}}(\gamma)=\sinh [(1+j) \gamma / \delta] / \sinh [(1+j) d / 2 \delta] \\
& \underline{\mathrm{g}}(\gamma)=\cosh [(1+j) \gamma / \delta] / \cosh [(1+j) d / 2 \delta]
\end{aligned}
$$

where $d$ is the plate thickness and $\gamma$ is equal to zero at the midthickness of the plate.

The integral value $\underline{G}$ of $g$ will also be used in this paper (Fig. 1):

$$
\underline{\mathrm{G}}=\frac{1}{d} \int_{d} \underline{\mathrm{g}}(\gamma) \cdot d \gamma=\tanh [(1+j) d / 2 \delta] /[(1+j) d / 2 \delta] .
$$

If these particular variations in the sheets are accepted, the values of the tangential component of each field $\left(h_{\alpha}, b_{\alpha}, e_{\beta}\right.$ or $j_{\beta}$ ) on the surfaces of the sheets define it everywhere; we get for any tangential quantity $\underline{x}$

$$
\underline{\mathrm{x}}(\gamma)=\frac{1}{2}\left[\underline{\mathrm{x}}\left(\frac{d}{2}\right)+\underline{\mathrm{x}}\left(-\frac{d}{2}\right)\right] \cdot \underline{\mathrm{g}}(\gamma)+\frac{1}{2}\left[\underline{\mathrm{x}}\left(\frac{d}{2}\right)-\underline{\mathrm{x}}\left(-\frac{d}{2}\right)\right] \cdot \underline{\mathrm{f}}(\gamma) .
$$

\section{B. Homogenized Functions $\underline{\mathrm{X}}$ and $\underline{\mathrm{X}}$ for $\underline{\mathrm{x}}(\gamma)$}

If the function $\underline{x}$ is continuous at the limit between two sheets (typically the tangential magnetic field), we can build a new function $\underline{X}$, which is equal to $\underline{x}$ on these limits and varies linearly in the sheets [Fig. 2(a)]. Then we get

$$
\begin{aligned}
\underline{\mathrm{X}}(\gamma=0) & =\frac{1}{2}\left[\underline{\mathrm{x}}\left(\frac{d}{2}\right)+\underline{\mathrm{x}}\left(-\frac{d}{2}\right)\right] \quad \text { and } \\
\left(\frac{\delta \underline{\mathrm{X}}}{d}\right)^{i} & =\frac{\left[\underline{\mathrm{x}}\left(\frac{d}{2}\right)-\underline{\mathrm{x}}\left(-\frac{d}{2}\right)\right]}{d} \text { for sheet }(i)
\end{aligned}
$$

If $\underline{x}$ is not continuous at the limit (the typical example for such a noncontinuous function is the tangential electric field), it remains possible to built a continuous function $\underline{X}$ in respect to (5) and varying linearly between the centers of two consecutives sheets. The slope of $\underline{X}$ depends on the four limit values of $\underline{x}$ for 

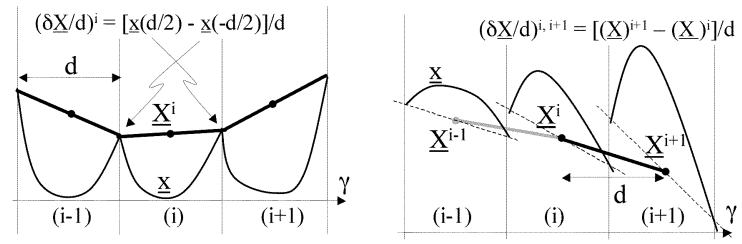

Fig. 2. Homogenized function $\underline{X}$ of (a) a continuous or (b) discontinuous function $\underline{\mathrm{x}}(\gamma)$, and values of their slopes.

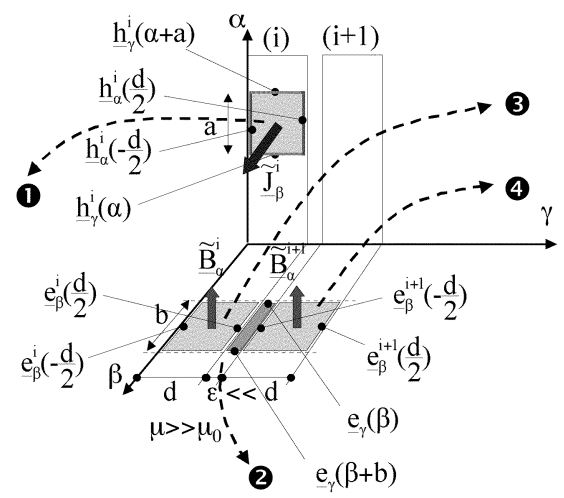

Fig. 3. Ampère's theorem and Lenz's law in the sheets.

these two sheets: (6) is no more valid [Fig. 2(b)]. $\underline{\mathrm{X}}$ is a possible homogenization of $x$.

We could also build a homogenized function $\underline{\widetilde{X}}$ from the mean value of $\underline{x}$ through the sheet

$$
\underline{\tilde{\mathrm{X}}}(\gamma=0)=\frac{1}{d} \int_{d} \underline{\mathrm{x}}(\gamma) \cdot d \gamma=\underline{\mathrm{G}} \cdot \underline{\mathrm{X}}(\gamma=0) .
$$

\section{Ampère's Law}

The homogenization requires to write again the Maxwell's equations - here, the Ampère's theorem and the Lenz's law-in an integral form, on a scale greater than (or equal to) the thickness $d$ of the sheets (Fig. 3, (1)):

$$
\left[\underline{\mathrm{h}}_{\alpha}\left(\frac{d}{2}\right)-\underline{\mathrm{h}}_{\alpha}\left(-\frac{d}{2}\right)\right]-d \cdot \lim _{a \rightarrow 0} \frac{\underline{\mathrm{h}}_{\gamma}(\alpha+a)-\underline{\mathrm{h}}_{\gamma}(\alpha)}{a}=\int_{d} \underline{\mathrm{j}}_{\beta}(\gamma) \cdot d \gamma \text {. }
$$

With notations (6) and (7), we get a partial differential equation in the direction parallel to the lamination, and a difference equation in the normal direction (the upper index $i$ marks the number of the concerned sheet)

$$
\left(\frac{\delta \underline{\mathrm{H}}_{\alpha}}{d}\right)^{i}-\frac{\partial \underline{\mathrm{H}}_{\gamma}}{\partial \alpha}=\underline{\widetilde{\mathbf{J}}}_{\beta}^{i}
$$

\section{Lenz's Law}

Starting with (Fig. 3, (3) for the sheet (i) and (4) for $(i+1)$ ):

$$
\begin{gathered}
{\left[\underline{\mathrm{e}}_{\beta}^{i}\left(\frac{d}{2}\right)-\underline{\mathrm{e}}_{\beta}^{i}\left(-\frac{d}{2}\right)\right]=j \omega \cdot \int_{d} \underline{\mathrm{b}}_{\alpha}^{i}(\gamma) \cdot d \gamma=d \cdot j \omega \cdot \underline{\widetilde{\mathrm{B}}}^{i}} \\
{\left[\underline{\mathrm{e}}_{\beta}^{i+1}\left(\frac{d}{2}\right)-\underline{\mathrm{e}}_{\beta}^{i+1}\left(-\frac{d}{2}\right)\right]=j \omega \cdot \int_{d} \underline{\mathrm{b}}_{\alpha}^{i+1}(\gamma) \cdot d \gamma=d \cdot j \omega \cdot \underline{\widetilde{\mathrm{B}}}^{i+1}}
\end{gathered}
$$

we can write

$\underline{\mathrm{e}}_{\beta}^{i}\left(\frac{d}{2}\right)=\underline{\mathrm{E}}_{\beta}^{i}+d \cdot j \omega \frac{\widetilde{\mathrm{B}}_{\alpha}^{i}}{2}$ and $\underline{\mathrm{e}}_{\beta}^{i+1}\left(-\frac{d}{2}\right)=-\underline{\mathrm{E}}_{\beta}^{i+1}+d \cdot j \omega \frac{\widetilde{\mathrm{B}}_{\alpha}^{i+1}}{2}$.
The Lenz's law written between the two iron sheets (Fig. 3, (2), where $\underline{\mathrm{e}}_{\gamma}$ is supposed to be zero in the sheets, but not between them) gives

$$
\left[\underline{\mathrm{e}}_{\beta}^{i}\left(\frac{d}{2}\right)-\underline{\mathrm{e}}_{\beta}^{i+1}\left(-\frac{d}{2}\right)\right]-\varepsilon \cdot \lim _{b \rightarrow 0} \frac{\underline{\mathrm{e}}_{\gamma}(\beta+b)-\underline{\mathrm{e}}_{\gamma}(\beta)}{b}=-j \omega \mu_{0} \int_{\varepsilon} \underline{\mathrm{h}}_{\alpha}(\gamma) \cdot d \gamma
$$

Then, defining the mean electric field in direction $\gamma$ by ${ }^{1}$

$$
(d+\varepsilon) \mathrm{E}_{\gamma}=\varepsilon \underline{\mathrm{e}}_{\gamma}
$$

neglecting the right-hand term in (13) and using (12), we get

$$
\begin{aligned}
& \underline{\mathrm{E}}_{\beta}^{i}-\underline{\mathrm{E}}_{\beta}^{i+1}+d . j \omega \frac{\left(\underline{\widetilde{\mathrm{B}}}_{\alpha}^{i}+\underline{\widetilde{\mathrm{B}}}_{\alpha}^{i+1}\right)}{2} \\
&+(d+\varepsilon) \lim _{b \rightarrow 0} \frac{\mathrm{E}_{\gamma}(\beta+b)-\underline{\mathrm{E}}_{\gamma}(\beta)}{b}=0 .
\end{aligned}
$$

$\varepsilon \ll d$ is the thickness of the insulating between two iron sheets. ${ }^{2}$ The Lenz's law can now be rewritten as a mixed partial differential/difference equation:

$$
-\left(\frac{\delta \underline{\mathrm{E}}_{\beta}}{d}\right)^{i, i+1}+\frac{\partial \underline{\mathrm{E}}_{\gamma}}{\partial \beta}=-j \omega \cdot\left(\underline{\widetilde{\mathrm{B}}}_{\alpha}\right)^{i, i+1} .
$$

\section{E. Homogenized Properties $\mu$ and $\underline{\sigma}$}

It remains to be written the phenomenological relations between the homogenized quantities. It can be seen that (3), (4), (5), and (7) lead to $\underline{\widetilde{X}}=\underline{X} \underline{G}$; using the relations between the local fields $\underline{\mathrm{b}}=\mu \underline{\mathrm{h}}$ and $\underline{\mathrm{j}}=\sigma \underline{\mathrm{e}}$ we get $^{3}$

$\underline{\widetilde{\mathrm{B}}}_{\alpha}=\underline{\mu}_{\alpha \beta} \cdot \underline{\mathrm{H}}_{\alpha}$ and $\underline{\widetilde{J}}_{\beta}=\underline{\sigma}_{\alpha \beta} \cdot \underline{\mathrm{E}}_{\beta}$ with $\underline{\mu}_{\alpha \beta}=\mu \cdot \underline{\mathrm{G}}$ and $\underline{\sigma}_{\alpha \beta}=\sigma \cdot \underline{\mathrm{G}}$

In the normal direction $\gamma$, we will simply write

$\underline{\widetilde{B}}_{\gamma}=\mu \cdot \underline{\mathrm{H}}_{\gamma}$ and $\underline{\widetilde{J}}_{\gamma}=0$; that means: $\underline{\mu}_{\gamma}=\mu$. and $\underline{\sigma}_{\gamma}=0$.

Consequently, the homogenized permeability and conductivity are defined as complex tensors: $\underline{\mu}$ and $\underline{\sigma}$.

\section{F. Homogenized Maxwell-Like Equations}

At this stage of our chain of reasoning, the homogenized fields and properties that we have defined, coupled with the shape functions $\underline{f}$ and $g$, are just another way to write the analytical solutions in the normal direction of the laminated stack. The size of the problem has been reduced to one unknown per sheet in the normal direction, and it no longer depends on the relative skin depth.

The homogenization principle itself consists in assuming that the homogenized fields are a continuum following - as a first step-(9) and (16) with [refer to Fig. 2(a) for $\underline{\mathrm{H}}_{\alpha}$ and Fig. 2(b) for $\left.\underline{E}_{\beta}\right]$

$$
\left(\frac{\delta \underline{\mathrm{H}}_{\alpha}}{d}\right)^{i} \rightarrow \frac{\partial \underline{\mathrm{H}}_{\alpha}}{\partial \gamma} \text { and }\left(\frac{\delta \underline{\mathrm{E}}_{\beta}}{d}\right)^{i, i+1} \rightarrow \frac{\partial \underline{\mathrm{E}}_{\beta}}{\partial \gamma}
$$

\footnotetext{
${ }^{1}$ That means that the circulations of the homogenized and actual fields $\underline{\mathrm{E}}$ and e are equal in the direction $\gamma$.

${ }^{2}$ Compared with $d$, the thickness $\varepsilon$ of the insulation will be neglected in this paper: It could easily be taken into account in the homogenized properties (11) and (12), which just become more complicated.

${ }^{3}$ As far as possible, the notations of paper [2] are used here. Even if it is not obvious, it can be shown that the term $\mathrm{F}_{\mathrm{R}}$ of paper [2] is equal to $1 / \underline{\mu}_{\alpha \beta}$ : Both approaches are consistent.
} 
and, more generally speaking, the Maxwell-like equations

$\nabla \times \underline{\mathrm{H}}=\underline{\widetilde{\mathrm{J}}} ; \nabla \times \underline{\mathrm{E}}=-j \omega \cdot \underline{\widetilde{\mathrm{B}}} ; \nabla \cdot \underline{\widetilde{\mathrm{B}}}=0 ; \underline{\widetilde{\mathrm{B}}}=\underline{\mu} \cdot \underline{\mathrm{H}} ; \underline{\widetilde{\mathrm{J}}}=\underline{\sigma} \cdot \underline{\mathrm{E}}$.

Replacing a system of difference equations by a continuous one, we clearly do an approximation: however, this is necessary if we want to introduce the homogenization in standard FEM software, with meshes much larger than the thickness of the sheets. Will the method be more and more accurate, as far as the number of sheets increases? We will show that this condition is not sufficient to make the real solution compatible with the approximation done in (19).

\section{ENERGETIC INTERPRETATION}

In the real system, the losses are solely due to the eddy-currents, and depend on $\gamma$. For one sheet, the mean value of these losses ("macroscopic" active power density) is

$$
\begin{aligned}
\widetilde{\mathrm{p}}=\frac{1}{d} \int_{d} \underline{\mathrm{e}}_{\alpha}(\gamma) \cdot \underline{\mathrm{j}}_{\alpha}^{*}(\gamma) d \gamma & =\underbrace{\frac{\left|\underline{\mathrm{j}}\left(\frac{d}{2}\right)+\underline{\mathrm{j}}\left(-\frac{d}{2}\right)\right|^{2}}{4 \sigma d} \int_{d} \mathrm{~g}^{2} d \gamma}_{\mathrm{a}} \\
& +\underbrace{\frac{\left|\underline{\mathrm{j}}\left(\frac{d}{2}\right)-\underline{\mathrm{j}}\left(-\frac{d}{2}\right)\right|^{2}}{4 \sigma d} \int_{d} \mathrm{f}^{2} d \gamma}_{b} .
\end{aligned}
$$

After some (difficult) calculations, we can express it from the homogenized fields

$$
\widetilde{\mathrm{p}}=\operatorname{Re}(\underbrace{\underline{\mathrm{E} \cdot \underline{\widetilde{\mathrm{J}}}}}_{\mathrm{a}}-\underbrace{j \omega \underline{\mathrm{H}} \cdot \underline{\widetilde{\mathrm{B}}}^{*}}_{b}) \quad\left[\mathrm{W} / \mathrm{m}^{3}\right] .
$$

In the same way, the imaginary part in (22) can be expressed as the mean value of $-\omega \underline{h b}^{*}$; it represents the macroscopic reactive power density

$$
\widetilde{\mathrm{q}}=\frac{1}{d} \int_{d}-\omega \underline{\mathrm{h}}_{\beta}(\gamma) \cdot \underline{\mathrm{b}}_{\beta}^{*}(\gamma) d \gamma=\operatorname{Im}\left(\underline{\mathrm{E}} \cdot \underline{\widetilde{\mathrm{J}}}^{*}-j \omega \underline{\mathrm{H}} \cdot \underline{\widetilde{\mathrm{B}}}^{*}\right) .
$$

This confirms the relevance of our choices for the homogenized quantities and properties.

The first term (a) in (21) and (22) is linked to the even part of the local current density $\mathrm{j}(\gamma)$; it will be represented in the continuous model by the losses of the homogenized current density $\widetilde{J}$, which could be regarded as the macroscopic current density. The term (b) is linked to the odd part of the local current, which cannot be observed on a macroscopic level; it will be represented by the losses due to the difference of phase between the homogenized fields $\underline{\widetilde{B}}$ et $\underline{H}$.

\section{Testing THE MODEL}

Manifold tests could be managed in 2-D or 3-D to compare FE solutions obtained either with the "sheet-by-sheet" description of a laminated region, or with the homogenized model.

\section{A. Effect of the Homogenization Approximation}

In this section, we will present some tests pointing out the accuracy of the homogenized equations (17), (18), and (20) in comparison with the real solution of the difference equations: the aim is to show the effect of the approximation (19).

We define a simple lamination stack configuration with nonzero mean field and current in the sheets (Fig. 4). The test device is excited on its front and back faces by a harmonic

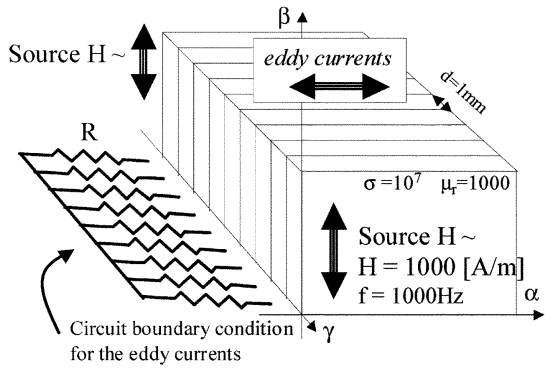

Fig. 4. Definition of the test device.

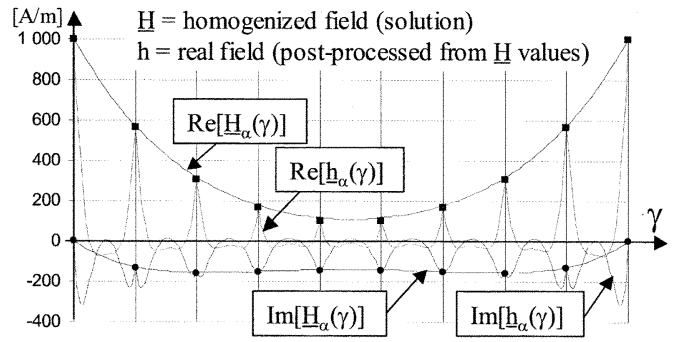

Fig. 5. Comparison of the homogenized partial differential equation solution (lines) with the exact solution of the difference equation (points) for the homogenized magnetic field $\underline{H}$, and corresponding local variations of $\underline{\mathrm{h}}(\mathrm{R}=50)$.

tangential magnetic field in direction $\beta$. The global current (direction $\alpha$ ) in each sheet is controlled by circuit equations, equivalent to resistances $\mathrm{R}$ (all identical and connected to a common point) ${ }^{4}$

The exact solutions for this "1.5-D" problem exist, for the "real" stack (solution of a difference equation, easily obtained with a simple spreadsheet), as for the homogenized equivalent system: in this case, the homogenized system of equations can be written in terms of magnetic field:

$$
\frac{\partial^{2} \underline{H}_{\alpha}(\gamma)}{\partial \gamma^{2}}-\frac{j \omega \underline{\sigma} \underline{\mu}}{1+R d \underline{\sigma}} \cdot \underline{H}_{\alpha}(\gamma)=0
$$

It is, therefore, possible to show the error due to the homogenization itself, independently of any FE formulation.

For the results presented in Fig. 5, we chose $\mathrm{R}$ to obtain nonzero global currents in each sheet, however, a solution very different from that of the simple short circuit. The comparison shows how the solution of the homogenized equations represents the real laminated device.

This practically perfect agreement was also verified for all the configurations we have processed, as long as the real solution does not vary too suddenly in the first sheets. We present such a case in Fig. 6. Note that the resultant nonlocal skin effect [related to (24)] depends on the external conditions (R) and not directly on the skin depth ( $\delta$ is the same for cases of Fig. 5 and Fig. 6): it will not be possible to predict the validity of the homogenized formulation using intrinsic criteria (frequency, conductivity, permeability).

\section{B. Test of the 3-D Finite Element Implementation}

Two 3-D FE models have been developed and applied to this study of lamination stacks with nonzero global currents. Both

\footnotetext{
${ }^{4}$ For $\mathrm{R} \rightarrow \infty$, each sheet is completely insulated from the others; the solution is the same for all the sheets, with a zero global current. For $\mathrm{R}=0$ (short circuit) the lamination has no more effect, and the solution is that of a solid conductor; as a consequence, the global current in each sheet is not zero.
} 


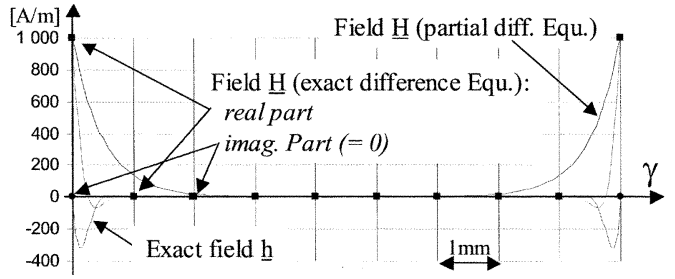

Fig. 6. Example of nonconvergence of the partial differential equation to the solution of the difference equation $(\mathrm{R}=0)$.

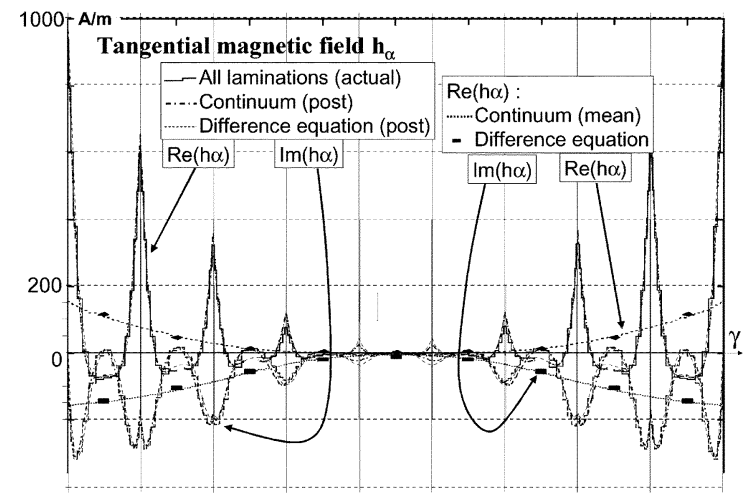

Fig. 7. Tangential field values: 3 -D FE solutions (actual + continuum) and comparison with the exact solution obtained by the difference equation.

models are based on the magnetic vector potential magnetodynamic formulation.

The first model directly considers the stack as a set of massive conductors separated by insulating layers [3]. In view of the large number of laminations encountered in iron cores, such a model is generally unfeasible and is only applied here to simple local problems for validation.

The second model considers the lamination stack as a source conductor through a precalculated current density distribution corresponding to the eddy currents in the laminations, as well as the actual distribution of the magnetic flux density. Both current density and magnetic flux density distributions in each lamination can be obtained by considering the analytical solution of the Maxwell equations governing the eddy currents while neglecting their fringing effects. Such distributions have been considered in [2] for zero global currents and can be expressed in terms of the mean magnetic flux density. They can be extended for nonzero global currents constraints, which aims at adding dependence with the mean current density. The actual current and magnetic flux density distributions are then integrated in the $\mathrm{FE}$ formulation, as done in [2], leaving their mean values as the unknowns of the problem. In this way, the lamination stack is transformed into a continuum, equivalent to what has been presented in Section II.

To lighten the computational work for the test problem and to focus on the main part of interest, the studied domain is reduced to the lamination stack. The magnetomotive force is fixed as a weak global quantity associated with the global magnetic flux, of which the distribution is obtained through the FE analysis [3]. A nonzero flux crossing the lateral boundary of the stack is defined through the use of a surface scalar potential associated with the surface magnetic vector potential [4]. This scalar po-

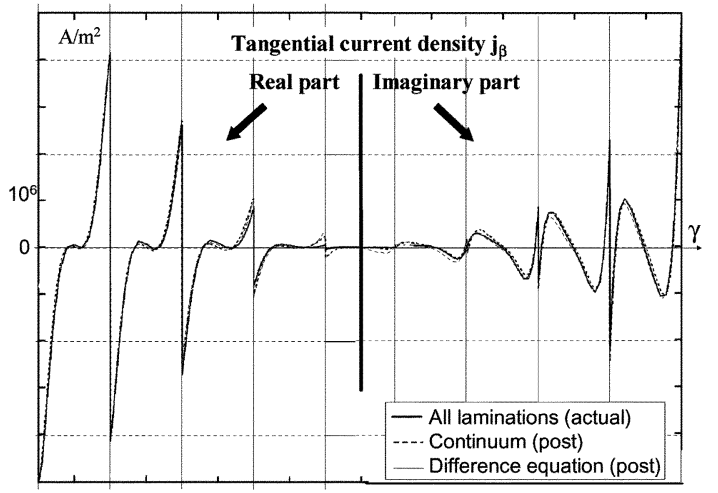

Fig. 8. Local values of the current density. Comparison between FEM (actual + continuum) and exact solution (difference equation).

tential is multivalued and has to undergo discontinuities along lines making the lateral surface simply connected. Such constraints define the boundary conditions.

The direct model needs to mesh each lamination separately, while the mesh is coarser for the continuous model. We consider conductive volume regions in contact with the lamination ends (direction $\alpha$ ), in order to obtain a solution with a nonzero homogenized current density. Both FE solutions are in perfect agreement, and coincide also perfectly with the corresponding 1.5-D difference equation solution (Figs. 7 and 8).

\section{CONCLUSION}

The tests of the homogenization have been done separately from its numerical FE implementation. The limit of validity has been shown: it runs perfectly only if the global skin effect is not prevailing inside the external sheets. Two ways could be then investigated to deal with this situation: either to solve directly the difference equations (9) and (16) in the direction perpendicular to the lamination, or to apply the surface impedance method to the external sheets [5], [6], in association with the homogenization technique for the other sheets.

The implementation of the homogenized equations in FE software does not pose any technical problem, because the form of the classical equations is preserved. Only complex tensors have to be used for the material properties, instead of real numbers; the post-processing may be modified, if local values are required in the laminations.

\section{REFERENCES}

[1] K. Hollaus and O. Biro, "Estimation of 3-D eddy-currents in conducting laminations by an anisotropic conductivity and a 1-D analytical model," COMPEL, vol. 18, no. 3, pp. 496-499, 1999.

[2] P. Dular et al., "A 3-D magnetic vector potential formulation taking eddy currents in lamination stacks into account," IEEE Trans. Magn., vol. 39, pp. 1147-1150, May 2003.

[3] _ "Modeling of thin insulating layers with dual 3-D magnetodynamic formulations," IEEE Trans. Magn., vol. 39, pp. 1139-1142, May 2003.

[4] — - "Complementary finite element magnetodynamic formulations with enforced magnetic fluxes," COMPEL, vol. 18, no. 4, pp. 656-667, 1999.

[5] L. Krähenbühl and D. Muller, "Thin layers in electrical engineering," IEEE Trans. Magn., vol. 29, pp. 1450-1455, Mar. 1993.

[6] H. Igarashi, A. Kost, and T. Honma, "A 3-D analysis of magnetic fields around a thin magnetic conductive layer using vector potential," IEEE Trans. Magn., vol. 34, pp. 2539-2542, Sept. 1998. 